\section{PICTURE QUIZ}

Journal of the Ceylon College of Physicians, 2013, 44, 47

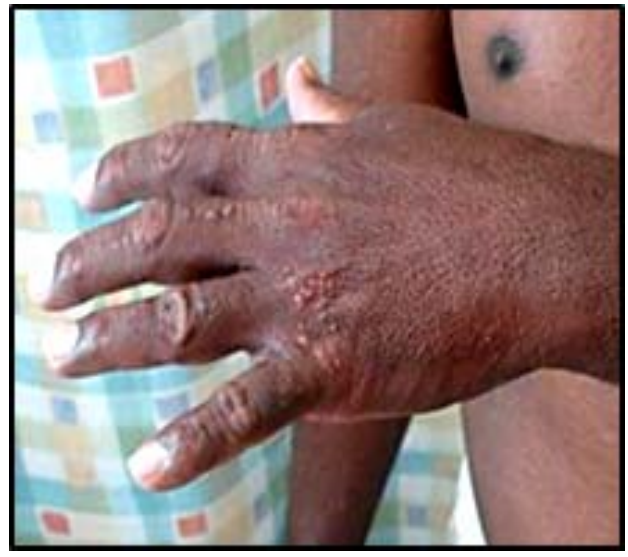

1. This 48yr old patient was found to have a "M" band in serum protein electrophoresis. He was found to have this abnormality in the skin. What is the diagnosis?

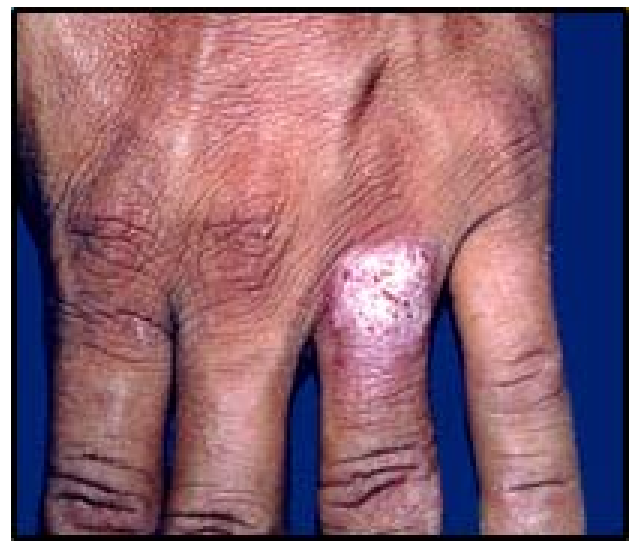

3. A nursing officer complains of this rash. He had been healthy apart from pulmonary tuberculosis 6yrs back. What is the diagnosis?

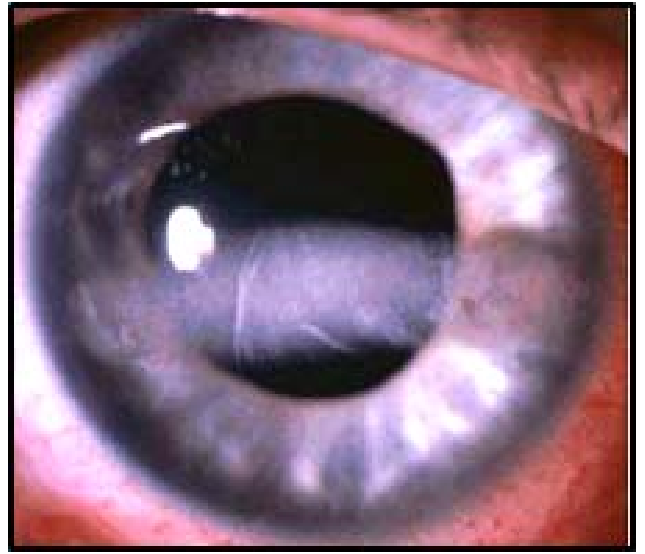

2. A 59-yr-old patient complains of reduced vision. He was found to have this abnormality in the eye? What is the diagnosis?

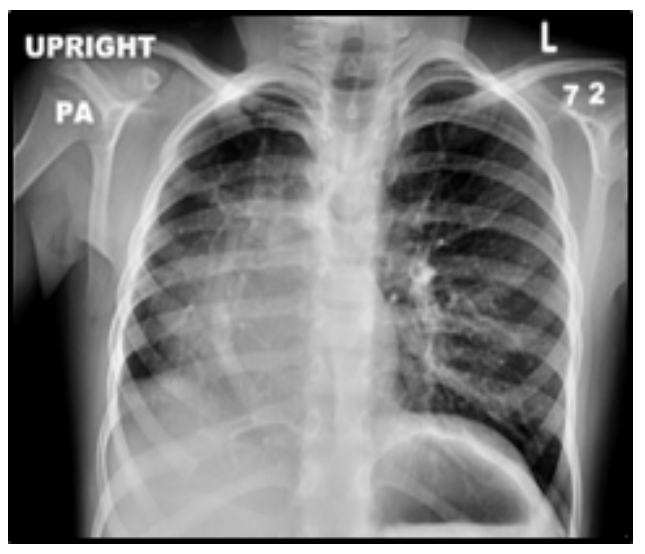

4. This chest X-ray is from a young boy with a VSD and a $\mathrm{GU}$ tract anomaly. What is the probable cause for this $\mathrm{X}$-ray appearance?

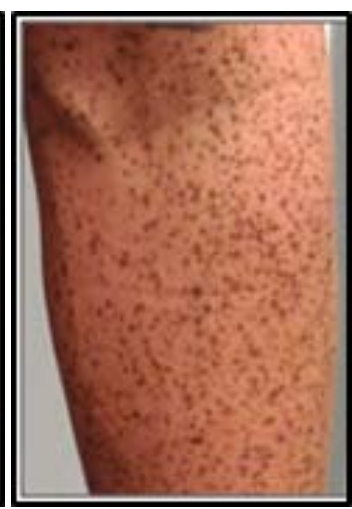

5. This 32-yr-old man was investigated for a systolic murmur. He has undergone surgery for cryptorchidism in childhood. What is the diagnosis? 


\section{ANSWERS TO PICTURE QUIZ:}

\section{Q1. Scleromyxedema}

Scleromyxedema (SM), also known as generalized lichen myxedematosus (LM), is a cutaneous mucinosis. Clinically, SM appears as a generalized eruption of $2 \mathrm{~mm}$ to $3 \mathrm{~mm}$ waxy lichenoid (flattopped) papules, often in a linear arrangement; lesions are most common on the hands, elbows, forearms, upper trunk, face, and neck. These lichenoid lesions coalesce, leading to induration of the underlying tissue that resembles scleroderma. Typical involvement of the glabella with deep longitudinal furrows can be reminiscent of leonine facies and a characteristic elevated rim with central depression can be seen on the proximal interphalangeal joints ("doughnut sign").

SM is associated most frequently with monoclonal gammopathy and other systemic disorders that may result in death. Systemic manifestations may involve the cardiovascular, gastrointestinal, pulmonary, rheumatologic, and central nervous systems. $83 \%$ of cases have an associated paraproteinemia and a minority of these patients develop plasma cell dyscrasia or MM (10\%). Other associated diseases include Waldenstrom's macroglobulinemia as well as Hodgkin's and non-Hodgkin's lymphomas.

\section{Q2. Band keratopathy}

Band keratopathy is characterized by the appearance of a band across the central cornea, formed by the precipitation of calcium salts on the corneal surface (directly under the epithelium). This form of corneal degeneration can result from a variety of causes, either systemic or local, with visual acuity decreasing in proportion to the density of the deposition. Hypercalcaemia, discoid lupus erythematosus and tuberous sclerosis are systemic conditions associated with band keratopathy.

\section{Q3. Tuberculosis verrucosa cutis}

Also known as "Lupus verrucosus, "Prosector's wart," and "Warty tuberculosis" is a rash of small, red papular nodules in the skin that may appear 2-4 weeks after inoculation by Mycobacterium tuberculosis in a previously infected and immunocompetent individual.

It is so called because it was a common occupational disease of prosectors, the preparers of dissections and autopsies. Reinfection by tuberculosis via the skin, therefore, can result from accidental exposure to human tuberculous tissue in physicians, pathologists and laboratory workers.

\section{Q4. Scimitar syndrome}

Chest x-ray demonstrates a small right lung with ipsilateral mediastinal shift. A tubular structure is seen paralleling the right heart border in the shape of a Turkish sword ("scimitar").

Scimitar syndrome or pulmonary venolobar syndrome is a rare congenital heart defect characterized by anomalous venous return from the right lung (to the systemic venous drainage, rather than directly to the left atrium). Patients develop heart failure due to the left to right shunt. Associations include ASD, VSD, PDA, vertebral anomalies, pulmonary sequestration, diaphragmatic anomalies and GU tract abnormalities.

\section{Q.5 LEOPARD Syndrome}

LEOPARD syndrome (Cardiocutaneous syndrome, Gorlin syndrome II or Moynahan syndrome) is a rare autosomal dominant multisystem disease. The name of the condition is a mnemonic, originally coined in 1969 as the condition is characterized by some of the following seven conditions, the first letters of which spell LEOPARD.

$\mathrm{L}$ : Lentigines

E : ECG anomalies (Conduction defects)

$\mathrm{O}$ : Ocular hypertelorism

$\mathrm{P}$ : Pulmonary stenosis (sometimes aortic stenosis and MVP)

A : Abnormal genitalia

$\mathrm{R}$ : Retarded growth

D : Deafness

Compiled by Dr Nilanka Perera, Department of Medicine, University of Sri Jayewardenepura, Sri Lanka. 